\section{Cahiers de Narratologie}

Analyse et théorie narratives

$10.1 \mid 2001$

La voix narrative

\title{
Le silence dans Amado Monstruo et El castillo de la carta cifrada de Javier Tomeo
}

\section{Mor Gaye}

\section{(2) OpenEdition}

1 Journals

\section{Electronic version}

URL: http://journals.openedition.org/narratologie/6981

DOI: 10.4000/narratologie.6981

ISSN: 1765-307X

\section{Publisher}

LIRCES

\section{Printed version}

Date of publication: 1 January 2001

Number of pages: 495-502

ISBN: 2914561032

ISSN: 0993-8516

\section{Electronic reference}

Mor Gaye, "Le silence dans Amado Monstruo et El castillo de la carta cifrada de Javier Tomeo", Cahiers de Narratologie [Online], 10.1 | 2001, Online since 20 November 2014, connection on 24 February 2021. URL: http://journals.openedition.org/narratologie/6981 ; DOI: https://doi.org/10.4000/narratologie. 6981 


\title{
LE SILENCE DANS AMADO MONSTRUO ET EL CASTILLO DE LA CARTA CIFRADA DE JAVIER TOMEO
}

\author{
Mor GAYE \\ Université Paul Valéry Montpellier III
}

Si l'on cherche à définir notre conception du silence, elle est proche de la définition que lui donne Marc de Smedt : «Le silence, c'est l'envers de la façade, l'envers du masque, la face cachée de la personne. Le tremplin du mot $\gg 1$. Cette conception du silence appliquée à Amado monstruo et $E l$ castillo de la carta cifrada ${ }^{2}$ de Javier TOMEO, laisse supposer qu'un non-dit à l'origine du tumulte verbal des personnages se trouve sous-jacent au texte. On s'attachera à relever l'importance de ce silence, de ce non-dit, authentique vérité secrète non avouée, mais perceptible dans les discours des protagonistes de ces deux œuvres. La fonction du silence de l'écriture <<subjectiviste > q qui sature les discours en usant et en abusant de la fonction phatique montre la propension à la parole, au bavardage des personnages tomesques dont l'unique objectif est de taire leur monstruosité et leur solitude. La fonction première de cette écriture consiste donc à combattre le silence au moyen du bavardage. Nous remarquerons aussi au cours de notre étude et en premier lieu que le silence visible visant à décrire le comportement des protagonistes comme le dialogue de pantomimes ainsi que les nombreux déraillements du récit contribuent à la mise en silence de l'authentique diégèse : la monstruosité, la solitude synonymes du silence auquel nous venons de faire allusion.

1 «Les signes de la communication», Éloge du silence, Albin Michel, 1986, p. 49.

2 Javier TOMEO, Amado monstruo, éd. Anagrama, Barcelona, 1985.

- El castillo de la carta cifrada, ed. Anagrama, Barcelona, 1988. 
Amado monstruo de J. Tomeo pose une situation narrative où les deux interactants sont engagés dans l'échange verbal. Il s'agit du demandeur d'emploi : Juan D. et du chef du personnel d'une banque: Krugger. L'entretien d'embauche, curieusement, ne porte pas sur le poste de vigile mais sur une obsession commune des deux personnages : leur mère. Juan, à trente ans, tente d'échapper à l'amour d'une mère étouffante et Krugger vit toujours dans le souvenir étouffant d'une mère morte accidentellement. Le récit est la narration d'un dialogue rapporté à la première personne du présent par le narrateur Juan, lequel présent dans le bureau du banquier lui raconte le différend qui l'oppose à sa mère.

L'appartement de la mère est le siège d'un affrontement verbal axé sur une dualité temporelle. Le dialogue belliqueux est animé par deux projets contraires : celui du fils de se lancer dans un projet d'édification de l'avenir et celui de la mère de vivre dans la nostalgie du passé. Ce décalage temporel explique le désaccord verbal de ce couple : le fils se plaint d'être enfermé dans un monde caduc (74) et la mère gémit de ne pas avoir de lendemain (76). S'installe alors dans le récit « un dialogue de sourds » (76). Chaque personnage s'enferme dans la parole pour crier son désaccord. Le langage, loin d'être un moyen de communication, devient un instrument verbal de mésentente. Les deux personnages sont ainsi tournés vers le passé et projetés dans le futur. Après avoir adopté des indifférences feintes, dialogué dans le silence au moyen des pantomimes (28), (43), (44), le couple, par un pacte tacite (28), finit par observer un répit. Dans cette ambiance muette, l'appartement est la scène du dialogue de l'incommunicabilité passant par le silence : "Me quedé pues callado y ella volvió a equivocarse. Interpretó mal mi silencio, por aquello de quien calla otorga (30)/Puse mi vaso boca abajo y ella sin darse cuenta, vertió el vino sobre el mantel (31). La communication se trouve à la frontière de l'échange verbal et de l'échange sans parole. L'entente dans la parole et dans le silence s'avère être impossible. Le récit se focalise continuellement autour de ce dialogue de la pantomime, mais derrière cette confrontation, source d'équivoque, se tient un autre silence tenu par une parole secrète qui parle cependant pour dire son intolérable absence : le non-dit de la double 
monstruosité de Juan qui a six doigts à chaque main et de sa mère en tant que corps insolite « debe pesar alrededor de cien kilos » (27). Le lecteur averti pensera certainement que c'est cette anomalie anatomique et physique que le récit scelle réellement sous le nom de pacte tacite (28). Ce mutisme récurrent est destiné à brider la voix du silence qui peut à tout moment apparaître à la surface narrative. Quelque chose, tenu par une parole secrète, se joue et ni Krugger, ni le lecteur n'arrive à lui donner un sens. La structure même du récit contribue à accentuer ce jeu du silence et du mystère. Pour cela, il interrompt constamment sa linéarité, il est toujours désarticulé par un coup de frein qui l'oriente dans une nouvelle direction. Le glissement permanent d'une scène à une autre sous la houlette de la voix narrative permet au lecteur de découvrir que le dialogue de l'entrevue est biaisé. Il est interrompu soit par le banquier, soit par un événement inattendu : (28), (55), (88). On arrive à se trouver quelque peu désorienté par la fréquence du dialogue distordu. Mais les traces de cette discontinuité sont aisément retrouvées par Krugger, fin enquêteur et jouant le Hercule Poirot : «me pide, sin embargo, que ponga un poco de orden en el relato » (21). Il s'opère alors une reconstruction du récit dépouillé de ses différentes digressions (description des scènes antérieures, échappées du banquier dans le passé, de ses moments de rêve). Pour ce faire, l'enquêteur, à l'instar du marquis, utilise la technique du flash back cinématographique : «Hagamos como en el cine, volvamos atrás la película y vayámonos al principio » $(C C C, 85)$. L'écriture empiète sur elle-même, opère une marche à contre-courant et par la fréquence des analepses centrées sur la réorganisation chronologique des événements narrés (20-22-33), efface les quelques soupçons de l'innommable (le meurtre de Krugger qui a tué sa mère de fort belle manière, en la faisant glisser sur « l'hispanique légume »: les pois chiches). Quelque chose est tu dans cette reconstruction du récit mais interpelle sans arrêt le lecteur et le narrateur. Il s'agit de ces impressions diffuses «vago presentimiento » (53-54), des soupçons balisés par des réflexions « in petto » : «me pregunto » $(15,33,40,56)$, « pienso » $(20,85)$, " $m e$ digo », (49), lesquelles épaississent le mystère lié au banquier sans réellement tenter de le forcer. Le pouvoir de 
communication des mots est fortement mis en doute et « le manque » valorisé en silence.

Le dialogue des personnages, se dessaisit, par conséquent, de sa fonction de transmission de sens. Il est une sorte d'insignifiance expressive et tend surtout vers un refus et une prudence du narrateur de s'engager dans une parole compromettante : "Procuro responder con brevedad y precisión, sin alargarme demasiado (8-9), «Me muestro, sin embargo, prudente y guardo silencio » (11), (52), (72). Si les personnages sont, ici, avares de paroles et cherchent refuge dans le mutisme, en retour, ils sont vite minés par l'angoisse du silence. Juan, comme Krugger cherchent désespérément la communication verbale : «y para romper el silencio [...] carraspeo » (54)/ «(Krugger) hablaba en general » (73), « se pierde en confusas disquisiciones » (65).

Dans ce jeu volontaire de mutisme et de parole, le dialogue devient hermétique et remplit sa fonction de mise en silence de la vérité secrète (la triple monstruosité occultée des trois personnages).

Les créatures tomesques vouent, cependant, un véritable amour pour le bavardage. Cette passion pour la parole prend des allures excessives dans El castillo de la carta cifrada. Le récit, tel un écheveau qui se dévide, s'autogénére constamment et pourrait être ce "monstruo que se engendra y nace de sí mismo » (35) dont parle le « je » locuteur. Le personnage cache tout en parlant l'indicible. Le sens de la vraie diégèse se trouve exilé vers une réalité où la parole devient abondante et mensongère. Parler revient à taire sa solitude, rien de plus.

Le silence de « l'écriture subjectiviste ${ }^{3}$ met en rapport l'acte de parler et l'angoisse latente permanente du récit.

3 On parle d' "écriture subjectiviste " [...] quand le discours accorde la primauté absolue à la fonction phatique [...] Cette fonction phatique qui s'exerce à l'oral par une plus grande richesse de moyens qu'à l'écrit est exploitée par le discours littéraire qui imite le bavardage [...] Elle atteint son apogée dans certains types de discours qui «parlent » sans rien dire. Comme le sifflement de l'individu et la parole enfantine dans une situation solitaire, le bavardage s'oppose à une certaine angoisse. [...] 

de la carta cifrada de Javier Tomeo

C'est bien cette angoisse voilée par une avalanche de paroles dont il s'agit dans $E l$ castillo.... Le personnage principal de cette œuvre, un marquis, écrit une lettre brouillée « un galimatías » (109) qu'il qualifie de hiéroglyphe (99) à un comte hypothétique (El Conde de X, Don Demetrio López del Costillar) d'un proche comté et dicte à son valet Bautista des recommandations pour transmettre ce message qui pourrait se perdre en chemin. Le problème du " qui parle ? » génère une situation ambiguë attestée par l'incise initiale du roman :-No se preocupe, Bautista, y deje de temblar-me dijo aquella mañana el señor Marqués - (9). Ici, c'est le serviteur Bautista qui répète " exactement $»^{4}$ à la forme directe ce que lui dit son maître . Ce qui nous amène à distinguer dans ce roman deux niveaux d'énonciation et deux statuts du discours. Le discours direct du marquis adressé à son valet Bautista, et la reprise de ce même discours par Bautista, dans un nouvel acte énonciatif dont le destinataire reste inconnu. Se pose alors un certain nombre de questions sur les identités de l'expéditeur, du destinataire et du messager. Ce « je » du récit ne refère-t-il pas au maître s'adressant à son valet ? Ou bien au valet s'adressant au destinataire de la lettre (le comte) ou tout simplement au lecteur? Le problème du «qui parle » s'obscurcit et nous rappelle que " le propre de l'écriture « est d'empêcher de jamais répondre à cette question: Qui parle? $»^{5}$ auquel nous ajoutons à qui parle t-il ?

Considérons, cependant, que le maître s'identifie à ce « je » épistolier. C'est à partir de ce choix que va s'orienter notre étude du silence.

L'échange épistolaire constitue, pour le maître, le seul moyen d'existence possible pour renoncer à l'obscurité,

Pierre VAN DEN HeUVel, Parole, mot, silence. Pour une poétique de l'énonciation, éd. José Corti, Paris, 1985, pp. 60-61.

${ }^{4}$ La crédibilité du style direct pourrait être défaillante selon Oswald Ducrot: "Que le style direct implique de faire parler quelqu'un d'autre, de lui faire prendre en charge des paroles, cela n'entraine pas que sa vérité tienne à une correspondance littérale, terme à terme ", Le dire et le dit, Les Éditions de minuit, Paris, 1984, p. 199.

5 Roland BARThes, $S / Z$, Seuil, Paris, 1970, p. 146, cité par C. Kerbrat-Orecchioni, L'énonciation, Armand Colin, Paris, 1997, p. 172 . 
meubler son existence et apaiser sa solitude : «No soy original en esto, lo reconozco, porque gracias al género epistolar, muchas personas condenadas al silencio y a la soledad soportaron su condición con menos fatiga. (CCC,30).

La solitude, ici, est consubstantielle au silence. La fuite du marquis se voit ainsi liée à la peur de la solitude et du silence. Mais, il arrive que la solitude tue dans le récit étouffe le personnage. Son désespoir se tempère alors dans l'illusion de trouver le réconfort dans une vie ténébreuse : "En las tinieblas [...] reina siempre una misteriosa grandeza » (28) ou amoureuse : "La soledad-me dije un día-He ahí mi gran refugio. $Y$ me acogí a ella como se entrega un adolescente a su primer amor. (CCC, 48).

C'est un remède de jouissance certaine, un remède efficace qui fait aimer au châtelain ce qui l'écrase. De là, naît, certainement, la mélancolie du personnage qui entonne dans son discours les chants les plus désespérés.

$\mathrm{Si}$ le lecteur observe de près la situation de communication du récit, il s'apercevra que le marquis parle au serviteur en lui dictant des recommandations lorsque celui-ci transmettra la lettre au comte et joue en même temps sur son contenu codé, sur son écriture. L'échange verbal repose sur un énoncé d'une série de conseils et la fréquence des expressions phatiques: "Me sigue usted, Bautista » (38)(41), sert de recadrement et de resituation au déraillement du discours prêt à rebondir. L'angoisse de la perte du contact provoque ainsi l'intensification de la fonction phatique. Ce souci phatique conduit dans certains passages du roman à sa propre hypertrophie. Quant à l'écriture de la lettre, son codage forme le noyau central du récit: « ¿somfundamentaleslasramasqueridocomde?»(38). Cette pratique de l'écriture de la lettre est un moyen de prolonger le contact entre le marquis (l'expéditeur) et le comte (le destinataire). Ce dernier, en dépit de sa noblesse, n'est pas instruit (43) et son ignorance égale son étroitesse d'esprit " congénitas limitaciones mentales» (32). Cette peinture négative du comte contribue à accentuer le temps de la lecture de la lettre. L'encodage prolonge ce contact «para leer una sola cuartilla [el Conde) necesitará por lo menos 
un par de horas " (16). L'artifice de la lettre sert de fonction phatique au discours. Le marquis retrouve le pouvoir enchanteur de la parole et de l'écriture pour conjurer le silence, taire sa solitude. Ce qui révèle que «l'écriture subjectiviste » dont la fonction d'information est subordonnée à la fonction phatique est une lutte contre le silence. La lettre est une grande parole proférée et écrite "mi carta es una sola e inmensa palabra " (16) et se présente comme une thérapie. Il faut parler, se purger des mots, faire sortir le monde de soi au moyen du verbe et compter sur son imagination : " la fantasía es una primavera eterna " (21). Le discours du maître par une pratique digressive (univers des insectes (2224,51-53), de procédés de répétition avec variations mène à une inépuisable amplification du récit (récurrence des questions rhétoriques « ¿Qué hacer ? ¿Negarse ? ¿Permanecer de pie ? ¿Sentarse ? » (56), multiplication des suppositions, des futurs de conjecture, jeu de substitution absurde d'un adjectif à un autre "fundamentales/necesarias " (39-40). Toutes ces stratégies narratives servent de fer de relance au discours ouvert à tous les possibles. Et c'est là, précisément, que réside le sentiment de péché du marquis : "Dios me perdone, por haber dado tantos rodeos para decir una cosa tan simple » (49). Un péché bien conscient mais vite réfuté "prefiero pecar por exceso que por defecto » (38). Le binôme antinomique « exceso » / « defecto », comme la fréquence des oppositions : "doncellez y preñez, no puede ser a la vez 》 (107), des jeux d'exhibition de la fausse lettre et d'occultation de l'authentique (109), expriment l'étroit rapport conflictuel entre le dit et le non-dit. Quelque chose est dit dans ce duel mais disparaît aussitôt nommé. Le lecteur tente alors de reconstituer cette part manquante. C'est souvent dans cette tension entre le dit «exceso » et le non-dit «defecto », la plénitude du mot «preñez » et sa vacuité «doncellez », qu'on réussit à pressentir l'innommable. La dualité semble loger dans les paradoxes exprimés par les contraires et laisse apparaître un locuteur qui tout en s'exprimant, avoue implicitement son péché mais se refuse à livrer entièrement «l'intime ».

Parler sans nommer l'innommable (CCC), se taire pour brider la voix du silence ou parler pour rompre le silence $(A M)$, les personnages tomesques sont ainsi des amants du 
silence, de la parole et des fugitifs du silence. Le récit dans ces deux œuvres reste toujours soumis à une tension permanente entre « la conversation et la sous-conversation $»^{6}$.

6 Nathalie SARRAUTE, l'Ère du soupçon, Gallimard, Paris, 1956, pp. 121-123. 